\title{
In Silico Screening Identified Novel Small-molecule Antagonists of PAC1 Receptor ${ }^{[}$
}

\author{
Ichiro Takasaki, Ai Watanabe, Masafumi Yokai, Yurie Watanabe, Daichi Hayakawa, \\ Ryota Nagashima, Mamoru Fukuchi, Takuya Okada, Naoki Toyooka, Atsuro Miyata, \\ Hiroaki Gouda, and Takashi Kurihara
}

\begin{abstract}
Department of Pharmacology, Graduate School of Science and Engineering (I.T., A.W., R.N.), Graduate School of Innovative Life Sciences (I.T., T.O., N.T.), Department of Molecular Neurobiology, Graduate School of Medical and Pharmaceutical Sciences (M. F.), and Department of Bio-functional Molecular Engineering, Graduate School of Science and Engineering (T.O., N.T.), University of Toyama, Toyama, Japan; Department of Pharmacology, Graduate School of Medical and Dental Sciences, Kagoshima University, Kagoshima, Japan (M.Y., A.M., T.K.); and Department of Analytical and Physical Chemistry, School of Pharmacy, Showa University, Tokyo, Japan (Y.W., D.H., H.G.)
\end{abstract}

Received September 30, 2017; accepted January 18, 2018

\section{ABSTRACT}

Pituitary adenylate cyclase-activating polypeptide (PACAP) and its receptors are present in the spinal dorsal horn and dorsal root ganglia, suggesting an important role of PACAP signaling systems in the modulation of spinal nociceptive transmission. Previously, we found that intrathecal injection of PACAP or maxadilan, a selective PACAP type I (PAC1) receptor agonist, induced transient aversive responses followed by a long-lasting mechanical allodynia in mice, suggesting that PACAP-PAC1 receptor systems are involved in chronic pain and that selective PAC1 antagonists may become a new class of analgesics. Although several PAC1 antagonists, such as PACAP 6-38, have been reported, all of them are peptide compounds. In the present study, we identified new small-molecule antagonists of the PAC1 receptor using in silico screening and in vitro/vivo pharmacological assays. The identified small-molecule compounds, named PA-8 and PA-9, dose dependently inhibited the phosphorylation of CREB induced by PACAP in PAC1-, but not VPAC $_{1}$ - or $\mathrm{VPAC}_{2}$-receptor-expressing $\mathrm{CHO}$ cells. PA-8 and PA-9 also dose dependently inhibited PACAP-induced cAMP elevation with an $\mathrm{IC}_{50}$ of 2.0 and $5.6 \mathrm{nM}$, respectively. In vivo pharmacological assays showed that intrathecal injection of these compounds blocked the induction of PACAP-induced aversive responses and mechanical allodynia in mice. In contrast, the compounds when administered alone exerted neither agonistic nor algesic actions in the in vitro/vivo assays. The compounds identified in the present study are new and the first small-molecule antagonists of the PAC1 receptor; they may become seed compounds for developing novel analgesics.

\section{Introduction}

Pituitary adenylate cyclase-activating polypeptide (PACAP) was originally isolated from ovine hypothalamic extracts based on its ability to stimulate adenylate cyclase in rat anterior pituitary cell cultures (Miyata et al., 1989). PACAP exists as two variants, a 38-amino acid form (PACAP38) and a C-terminal truncated form (PACAP27) (Miyata et al., 1989, 1990), both of which share $68 \%$ homology with vasoactive intestinal polypeptide (VIP), suggesting that PACAP belongs to the VIP/ secretin/glucagon superfamily (Miyata et al., 1989, 1990;

This work was supported by a Grant-in-Aid for Scientific Research (C), Japan Society for the Promotion of Science (JSPS) [JSPS KAKENHI Grant No. JP26430067]; the Nakatomi Foundation; and the Center for Clinical and Translational Research of Kyushu University [Grant No. A80].

https://doi.org/10.1124/jpet.117.245415.

S This article has supplemental material available at jpet.aspetjournals.org.
Vaudry et al., 2009). Three distinct G-protein-coupled receptors mediate the actions of PACAP and VIP. The PACAP type I (PAC1) receptor, which is coupled mainly to adenylate cyclase/ protein kinase A, binds the two forms of PACAP with high affinity and selectivity. $\mathrm{VPAC}_{1}$ and $\mathrm{VPAC}_{2}$ receptors, which are also primarily coupled to adenylate cyclase, can bind both PACAP and VIP with similar affinities (Dickson and Finlayson, 2009; Vaudry et al., 2009).

PAC1 receptor is particularly abundant in the central nervous system, including the spinal dorsal horn (Dickson and Finlayson, 2009; Vaudry et al., 2009), where PACAPimmunoreactive fibers are also considerably localized (Moller et al., 1993; Dun et al., 1996). These fibers are thought to be predominantly primary afferents in origin, because capsaicin treatment induces the release of PACAP from rat spinal cord (Zhang et al., 1997; Sakashita et al., 2001) and a significant decrease in the number of PACAP-immunoreactive nerve

ABBREVIATIONS: AR, aromatic; BNST, bed nucleus of the stria terminalis; $\mathrm{CHO}$, Chinese hamster ovary; CREB, cAMP response element binding protein; 3D, three-dimensional; EC, extracellular; HBD, hydrogen bond donor; HP, hydrophobic; PACAP, pituitary adenylate cyclase-activating polypeptide; PAC1, pituitary adenylate cyclase-activating polypeptide type I; TBST, Tris-buffered saline/Tween 20; VIP, vasoactive intestinal polypeptide. 
fibers within the spinal cord (Moller et al., 1993). Furthermore, PACAP mRNA/immunoreactivity is localized primarily in calcitonin gene-related peptide or substance P-immunoreactive neurons in rat dorsal root ganglia (Moller et al., 1993; Mulder et al., 1994) and is markedly upregulated after peripheral nerve injury or inflammation (Zhang et al., 1995, 1996, 1998; Jongsma Wallin et al., 2003; Mabuchi et al., 2004). Several lines of evidence indicate that PACAP and its receptor systems could play an important role in the modulation of spinal nociceptive transmission. It has been reported that intrathecal injection of PACAP induces thermal hyperalgesia in mice (Narita et al., 1996; Ohsawa et al., 2002) and that formalininduced nociceptive responses are ameliorated by pretreatment with intrathecal PACAP 6-38, an antagonist of the PACAP receptor (Ohsawa et al., 2002; Davis-Taber et al., 2008). Formalin- or carrageenan-induced inflammatory pain and nerve injury-induced neuropathic pain have been found to decrease in mice deficient in PACAP and/or the PAC1 receptor (Jongsma et al., 2001; Mabuchi et al., 2004; Sándor et al., 2010).

Recently, we demonstrated that a single intrathecal injection of PACAP or maxadilan, a PAC1 receptor specific agonist (Uchida et al., 1998; Moro et al., 1999), induced transient nociceptive behaviors followed by a long-lasting mechanical allodynia in mice (Ohnou et al., 2016; Yokai et al., 2016), suggesting that spinal PACAP-PAC1 receptor systems play an important role in the modulation of spinal nociceptive transmission and induction of chronic pain. Our results, together with the previous studies, indicate that the PAC1 receptor has great potential as a drug target for treating both inflammatory and chronic neuropathic pain. Although several PAC1 receptor antagonists, such as PACAP 6-38, M65, and max.d.4 (Robberecht et al., 1992; Uchida et al., 1998; Moro et al., 1999), are used as pharmacological tools, all of them are peptide compounds. They are not practical as therapeutic agents because of their half-lives, lack of oral bioavailability, and difficulty in penetrating the blood-brain barrier. Therefore, the development of specific nonpeptide antagonists of the PAC1 receptor has been desired. However, to date, there have been no reports on the development of such PAC1 receptor antagonists.

In the present study, we attempted to identify potential antagonists of the PAC1 receptor using in silico screening followed by in vitro/vivo pharmacological assays. Here we report the development of novel small-molecule antagonists of the $\mathrm{PAC} 1$ receptor.

\section{Materials and Methods}

In Silico Screening. We performed a multifilter virtual screening protocol to explore candidate compounds for novel PAC1 receptor antagonists, as shown in Fig. 1. For this purpose, we used an NMR structure of the $N$-terminal extracellular (EC) domain of PAC1 receptor in complex with its peptide antagonist PACAP 6-38 (PDB ID: 2JOD) (Fig. 2A). When we examined the NMR structure, the residues Y22, V26, and R30 of PACAP 6-38 were found to be located on one side of its helical structure interacting with the PAC1 receptor, as shown in Fig. 2B. It has also been reported that a single point mutation of the residues Y22A, V26G, or R30A of PACAP 6-38 results in a drastic loss in affinity to the receptor (Sun et al., 2007), indicating that these residues play important roles in the antagonist activity of PACAP 6-38. Therefore, we explored compounds that were expected to interact with the PAC1 receptor in a manner similar to Y22, V26, and R30 of PACAP 6-38 (Fig. 2B).
Materials. PACAP (38-amino acid form), VIP, and PACAP 6-38 were purchased from Peptide Institute Inc. (Osaka, Japan). These drugs were made up as concentrated stock solutions in phosphatebuffered saline or physiologic saline, and stored at $-30^{\circ} \mathrm{C}$. An aliquot was diluted to the desired concentration in phosphate-buffered saline or artificial cerebrospinal fluid $\left(\mathrm{NaCl} 138 \mathrm{mM}, \mathrm{KCl} 3 \mathrm{mM}, \mathrm{CaCl}_{2}\right.$ $1.25 \mathrm{mM}, \mathrm{MgCl}_{2} 1 \mathrm{mM}$, D-glucose $1 \mathrm{mM}$ ) immediately before use.

Cell Culture. PAC1, $\mathrm{VPAC}_{1}$, and $\mathrm{VPAC}_{2}$ receptor-expressing $\mathrm{CHO}$ cells $\left(\mathrm{PAC} 1 / \mathrm{CHO}, \mathrm{VPAC}_{1} / \mathrm{CHO}\right.$, and $\mathrm{VPAC}_{2} / \mathrm{CHO}$ cells, respectively) were cultured in Dulbecco's modified Eagle's medium/F-12 (Invitrogen, Carlsbad, CA) supplemented with 10\% fetal bovine serum, $100 \mathrm{IU} / \mathrm{ml}$ penicillin, $100 \mu \mathrm{g} / \mathrm{ml}$ streptomycin, and $200 \mu \mathrm{g} / \mathrm{ml}$ G418 or $5 \mu \mathrm{g} / \mathrm{ml}$ blasticidin $\mathrm{S}$. The cells were propagated in a humidified $37^{\circ} \mathrm{C}$ incubator in $5 \% \mathrm{CO}_{2} . \mathrm{VPAC}_{1} / \mathrm{CHO}$ and $\mathrm{VPAC}_{2} /$ CHO cells were kindly provided by Dr. I. Tatsuno (Toho University, Tokyo, Japan).

Western Blot Analysis. Cells were seeded onto 12-well plates at $1 \times 10^{5}$ cells/well and incubated for 48 hours. After treatment with candidate compounds or $0.1 \%$ dimethylsulfoxide (vehicle control) for 30 minutes, the cells were stimulated with PACAP for 30 minutes.

Cellular materials were lysed in lysis buffer [150 mM NaCl, $1 \%$ NP-40, 10\% glycerol, protease inhibitor mix (Nacalai Tesque, Kyoto, Japan), and $20 \mathrm{mM}$ Tris-HCl (pH 7.4)]. The lysate (20 $\mu \mathrm{g}$ total protein per lane) was subjected to SDS-polyacrylamide gel electrophoresis (10\% gel) and then blotted onto PVDF membranes (GE healthcare, Buckinghamshire, UK). The membranes were blocked with Blocking One (Nacalai Tesque) at room temperature for 1 hour. The membranes were then incubated with primary antibodies in Tris-buffered saline $0.1 \%$ Tween 20 (TBST) and 5\% Blocking One at $4^{\circ} \mathrm{C}$ for $16-18$ hour, washed three times with TBST, and exposed to peroxidase-conjugated secondary antibody in TBST containing 5\% Blocking One at room temperature for 1 to 2 hours. Immunoreactive proteins were visualized by a luminescent image analyzer (LAS-1000 plus; Fujifilm, Tokyo, Japan) using an enhanced chemiluminescence detection system (Chemi-Lumi One L; Nacalai Tesque). The following primary antibodies were used; anti-phospho-CREB (Ser133) and anti-CREB (Cell Signaling Technology, Danvers, MA). The phosphorylation level of CREB was calculated as the ratio of the intensity of the band relative to that of total CREB protein in each sample.

Measurement of cAMP. cAMP-Glo Assay kit (Promega, Madison, WI) was used to measure intracellular cAMP levels according to the manufacturer's instructions. Briefly, the PAC1/CHO cells were

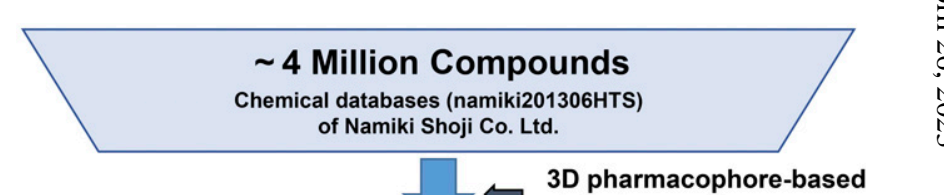

3D pharmacophore-based virtual screening

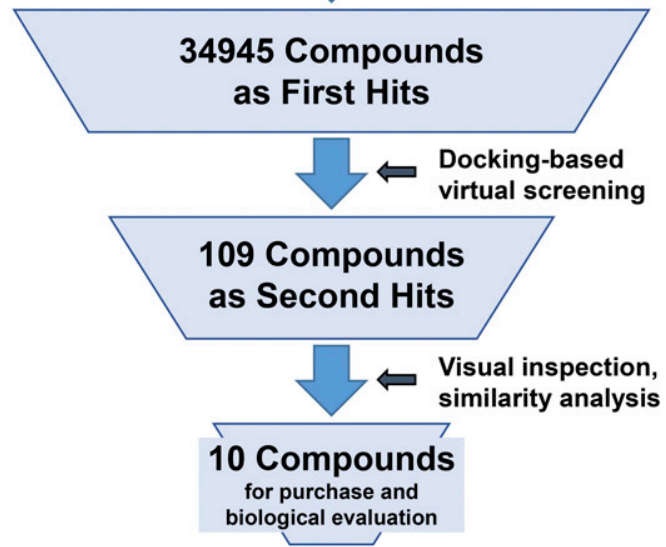

Fig. 1. Multifilter virtual screening. 

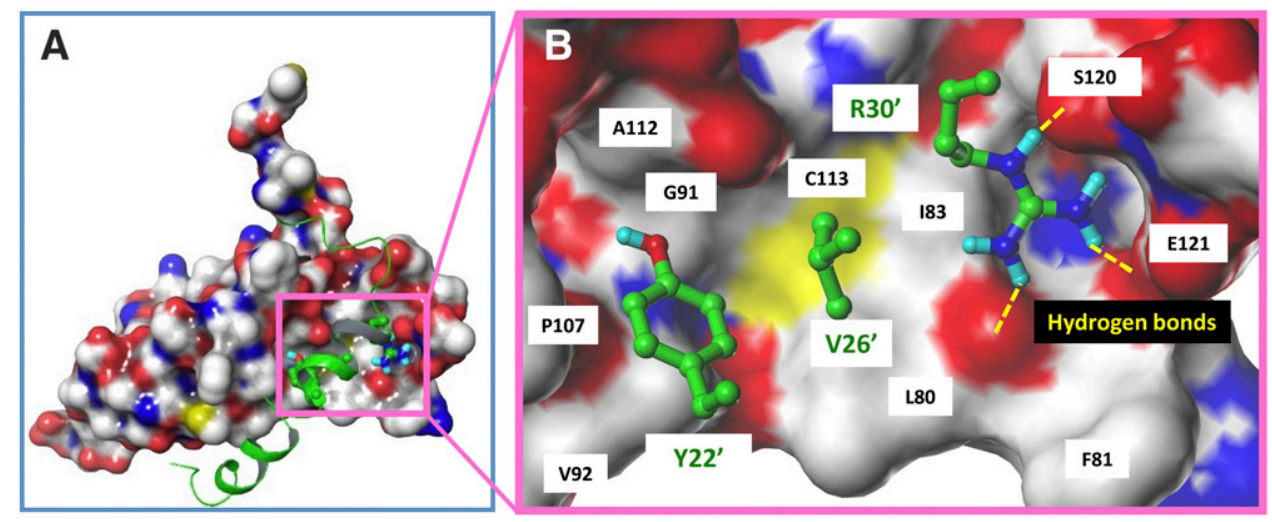

Fig. 2. (A) NMR structure of the $N$ terminal extracellular (EC) domain of PAC1 receptor in complex with PACAP 6-38 (green). (B) Interaction of Y22, V26, and R30 of PACAP 6-38 with PAC1 receptor. Hydrogen-bonding interactions are indicated by dashed yellow lines.

plated onto 96-well plates at $1 \times 10^{4}$ cells/well and incubated for 48 hours. After preincubation with the incubation medium [Dulbecco's modified Eagle's medium/F-12, $500 \mu \mathrm{M}$ isobutyl-1-methylxanthine, and $100 \mu \mathrm{M}$ 4-(3-butoxy-4-methoxy-benzyl) imidazolidone (Ro 20-1724)] for 1 hour, the cells were stimulated with PACAP ( $1 \mathrm{nM})$ for 1 hour. All candidate compounds were pretreated for 30 minutes before PACAP stimulation.

Animals. Male ddY mice (6 weeks old at the start of experiments) were purchased from Japan SLC Inc. (Shizuoka, Japan) and housed in standard polycarbonate cages (four mice per cage) under controlled temperature $\left(24 \pm 1{ }^{\circ} \mathrm{C}\right)$ and humidity $(55 \% \pm 10 \%)$ with a 12 -hour light-dark cycle (lights on at 0700 ) with food and water freely available. Mice were habituated to the animal facility for at least 1 week before experimentation. The animal experiments were approved by the Animal Care Committee of University of Toyama (approval no. 2014ENG-2) or Kagoshima University (approval no. MD 15011) and were conducted in accordance with the ethical guidelines for the study of experimental pain in conscious animals of the International Association for the Study of Pain (Zimmermann, 1983).

Intrathecal Injection and Behavioral Observation. Intrathecal injection was given in a volume of $5 \mu \mathrm{l}$ by percutaneous puncture through an intervertebral space at the level of the fifth or sixth lumbar vertebra, according to a previously reported procedure (Hylden and Wilcox, 1980).

Before intrathecal injection, mice were placed and habituated in a glass cylinder $(\phi 14 \times 18 \mathrm{~cm})$ with a filter paper at the bottom over 20 minutes. Immediately after intrathecal injection, the mice were placed again in the same glass cylinder, and the number of nociceptive behaviors consisting of licking, biting, and scratching directed toward the caudal part of the body was counted every 1 minute. The cumulative number of events was pooled over 5 -minute bins for 30 -minute period of observation and analyzed (Shimizu et al., 2004; Ohnou et al., 2016).

Mechanical sensitivity of the hind paw was evaluated with calibrated von Frey hairs (Stoelting Co., Wood Dale, IL) by measuring the tactile stimulus producing a 50\% likelihood of hind paw withdrawal response (50\% g threshold), which was determined using the up-down paradigm (Chaplan et al., 1994; Yokai et al., 2016).

All behavioral tests were done in double-blinded manner.

Statistical Analysis. Experimental data are expressed as mean \pm S.E.M. Single comparisons were made using Student's two-tailed unpaired $t$ test. One- or two-way analysis of variance followed by the Dunnett's test was used for multiple comparisons. For the analyses of mechanical thresholds, we employed the Mann-Whitney $U$ test for single comparisons or the Friedman test followed by the Steel test for multiple comparisons. $P<0.05$ was considered statistically significant.

\section{Results}

In Silico Screening. We performed a multifilter virtual screening protocol to explore candidate compounds for novel
PAC1 receptor antagonists, as shown in Fig. 1. In the first stage, we performed three-dimensional (3D) pharmacophorebased virtual screening. We generated 3D pharmacophore models based on Y22, V26, and R30 of PACAP 6-38 in the NMR structure (Fig. 2B), using the Unity module implemented in SYBYL-X Suite (Certara USA Inc., Princeton, NJ). As shown in Supplemental Fig. 1, we constructed two 3D pharmacophore models, both of which possess seven pharmacophore features. Models 1 and 2 differ in features on the hydroxyl group of Y22. Because the hydroxyl group is able to be a hydrogen bond acceptor as well as a donor, we defined hydrogen bond acceptor and donor features on the hydroxyl group of Y22 in models 1 and 2, respectively. As a result, model 1 comprises one aromatic (AR), two hydrophobic (HP), one hydrogen bond acceptor, and three hydrogen bond donor (HBD) features. In contrast, model 2 comprises one AR, two HP, and four HBD features. These two pharmacophore models were used as $3 \mathrm{D}$ structural queries for retrieving compounds from the chemical database (201306HTS) of Namiki Shoji Co. Ltd. (Tokyo, Japan) using the Unity module. The 3D coordinates of each molecule were generated with the CONCORD module of SYBYL, and the flex search protocol was performed to screen the database. We used a surface volume constraint based on a MOLCAD surface of the $N$-terminal EC domain of the PAC1 receptor. We extracted compounds satisfying the following criteria as "first hits": 1) matching with at least three of four hydrogen-bond related features (one hydrogen bond acceptor and three HBD features in model 1 and four HBD features in model 2) and 2) matching with at least two of three features consisting of one AR and two HP features. As a result, we obtained 25,737 and 9208 hits from models 1 and 2, respectively. In total, 34,945 hits were subsequently screened using protein structure-based virtual molecular docking (Fig. 1).

In the second stage, molecular docking was performed using the Schrödinger Suite 2013-2 (Schrödinger, LLC, New York, NY). First, the 3D structures of 34,945 hits were constructed using the LigPrep 2.7 program, and their protonation states were predicted using the Epik 2.5 program. The resulting conformers were used in the following docking calculations. The NMR structure of the $N$-terminal EC domain of the PAC1 receptor described above was used as a receptor for docking. We used a virtual screening workflow implemented in the Schrödinger Suite 2013-2. In this workflow, we first filtered hit compounds using Oprea's criteria of lead-likeness (Oprea et al., 2001). Then, first-stage docking was done in HTVS mode using GLIDE version 6.0 of the Schrödinger Suite 2013-2, and 
the top $10 \%$ poses were subjected to second-stage docking in SP mode. In the first and second docking calculations, we used hydrogen bond constraints, i.e., docking poses were generated to form hydrogen bonds with at least two residues among L80, S120, and E121 of the PAC1 receptor, which are known to form hydrogen bonds with R30 of PACAP 6-38 (Fig. 2B). Finally, binding free energies $\left(\Delta G_{b i n d}\right)$ for the top $10 \%$ poses derived from second-stage docking were estimated with the molecular mechanics generalized-born surface area method using the Prime 3.3 program. We extracted 109 compounds with $\Delta G_{b i n d}$ values of lower than $-50.00 \mathrm{kcal} / \mathrm{mol}$ as "second hits" (Fig. 1).

In the third stage, we first performed visual inspection of the second hits to select 50 compounds whose docking poses matched with several 3D pharmacophore features shown in Supplemental Fig. 1. The 50 selected compounds were finally clustered using similarity analysis based on their twodimensional structural fingerprints (Duan et al., 2010; Sastry et al., 2010) to reduce the number of virtual hits with which to proceed. The clustering was done with Canvas 1.7 of Schrödinger Suite 2013-2 (Fig. 1). As a result, we selected 11 compounds representing the cluster, of which nine (Supplemental Fig. 2, named PA-1 to 9) were commercially available. To find alternatives to two commercially unavailable compounds, similarity analysis of the 109 second hits was also performed. We found an alternative for one of unavailable compounds (Supplemental Fig. 2, PA-10) belonging to the same branch. Unfortunately, we could not obtain an alternative for the other unavailable compound, because it formed a one-member cluster. Therefore, 10 commercially available compounds were subjected to the following biologic evaluation.

Pharmacological Activity: Effects on PACAP-Induced CREB Phosphorylation and cAMP Elevation. To evaluate the antagonistic activity on the PAC1 receptors, we first examined the effects of the 10 identified candidate compounds (PA-1 to 10) on PACAP-induced CREB phosphorylation in PAC1/CHO cells (Fig. 3). PACAP 6-38 was used as a control antagonist. PACAP (10 pM to $1 \mu \mathrm{M})$ dose dependently induced CREB phosphorylation (data not shown), and a submaximal dose of PACAP (1 nM) was employed for pharmacological assays. Pretreatment with PACAP 6-38 (1 nM) significantly inhibited PACAP (1 nM)-induced CREB phosphorylation (Fig. 3 ). Among the 10 candidate compounds, we observed that PA-8, PA-9, and PA-10 (1 nM) significantly attenuated PACAP (1 nM)-induced CREB phosphorylation (Fig. 3). The inhibitory effects of PA-8 $(31.6 \% \pm 4.36 \%)$ and PA-9 $(35.7 \% \pm 5.56 \%)$ were comparable to or were marginally more potent than that of PACAP 6-38 $(42.4 \% \pm 5.68 \%)$.

Next, we examined the dose dependencies and receptor specificities of PA-8 and PA-9 (Fig. 4). In PAC1/CHO cells, PA-8 and PA-9 (10 pM to $10 \mathrm{nM}$ ) dose dependently inhibited PACAP (1 nM)-induced CREB phosphorylation. In $\mathrm{VPAC}_{1} /$ $\mathrm{CHO}$ and $\mathrm{VPAC}_{2} / \mathrm{CHO}$ cells, PACAP ( $1 \mathrm{nM}$ ) also induced CREB phosphorylation; however, this was not inhibited by PA-8 and PA-9 (10 pM to $10 \mathrm{nM})$ in these cells.

The functional potency of these compounds was also determined in PAC1/CHO cells using a cAMP assay (Fig. 5). PACAP (10 pM to $1 \mu \mathrm{M}$ ) dose dependently increased the intracellular cAMP level (data not shown) and a submaximal dose of PACAP ( $1 \mathrm{nM}$ ) was used for the cAMP assays. PA-8, PA-9, and PA-10 dose dependently inhibited the PACAP (1 nM)-induced increase in cAMP with an $\mathrm{IC}_{50}$ of 2.0, 5.6, and $33.1 \mathrm{nM}$, respectively (Fig. 5).

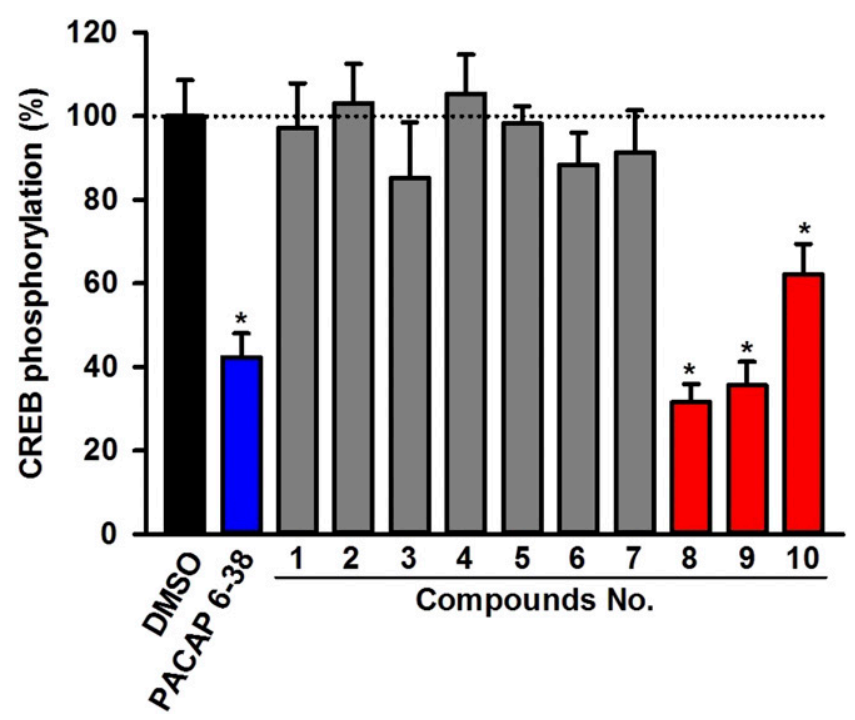

Fig. 3. Effects of 10 candidate compounds on the PACAP-induced phosphorylation of CREB in the $\mathrm{CHO}$ cells expressing PAC1 receptors. The cells were incubated with PACAP 6-38 (1 nM) or each compound (1 nM) for 30 minutes, then the cells were stimulated with PACAP (1 $\mathrm{nM}$ ) for 30 minutes and lysed. The lysates containing equivalent amounts of protein $(20 \mu \mathrm{g})$ were subjected to SDS-PAGE. Immunoblots were probed with specific antibodies that recognize phosphorylated form of CREB (pCREB) and total CREB. The data represent the mean \pm S.E.M. $(n=4$ to 5$)$. $* P<0.05$ vs. dimethylsulfoxide (DMSO) control.

We further investigated whether PA-8, PA-9, and PA-10 possessed any agonistic activity but found that these compounds (10 pM to $10 \mathrm{nM}$ ) did not induce CREB phosphorylation in $\mathrm{PAC} 1 / \mathrm{CHO}, \mathrm{VPAC}_{1} / \mathrm{CHO}$, or $\mathrm{VPAC}_{2} / \mathrm{CHO}$ cells (data not shown), suggesting that these compounds have no agonistic activities on PAC1, $\mathrm{VAPC}_{1}$, and $\mathrm{VPAC}_{2}$ receptors.

In summary, PA-8, PA-9, and PA-10 showed specific and potent antagonistic activities toward the PAC1 receptor and no detectable agonistic activity toward PAC1, $\mathrm{VPAC}_{1}$, and $\mathrm{VPAC}_{2}$ receptors. To the best of our knowledge, this is the first report on small-molecule antagonists of the PAC1 receptor.

Pharmacological Activity: Effects on PACAP-Induced Nociceptive Responses and Mechanical Allodynia. We previously demonstrated that intrathecal injection of PACAP $(100 \mathrm{pmol} / 5 \mu \mathrm{l})$ in mice induces transient aversive behaviors consisting of licking, biting, and scratching directed toward the caudal part of the body (Ohnou et al., 2016) followed by a longlasting mechanical allodynia of the hind paw (Yokai et al., 2016). Such aversive responses and mechanical allodynia were also induced by maxadilan, a PAC1 receptor-selective agonist, but not by VIP, a $\mathrm{VPAC}_{1} / 2$ receptor agonist, suggesting that intrathecal PACAP-induced nociceptive behaviors were mediated by the stimulation of spinal PAC1 receptors. Therefore, we examined the effects of PA-8, PA-9, and PA-10 on PACAPinduced aversive behaviors and mechanical allodynia (Fig. 6).

After a single intrathecal injection of PACAP, the number of aversive behaviors, such as licking and biting, gradually appeared at 3-5 minutes, reached a plateau within 15-20 minutes, and were sustained for more than 30 minutes (Fig. 6A), consistent with our previous report (Shimizu et al., 2004; Ohnou et al., 2016). Co-injection of PA-8, PA-9, or PA-10 (100 pmol each) with PACAP (100 pmol) potently attenuated the development of PACAP-induced aversive responses 


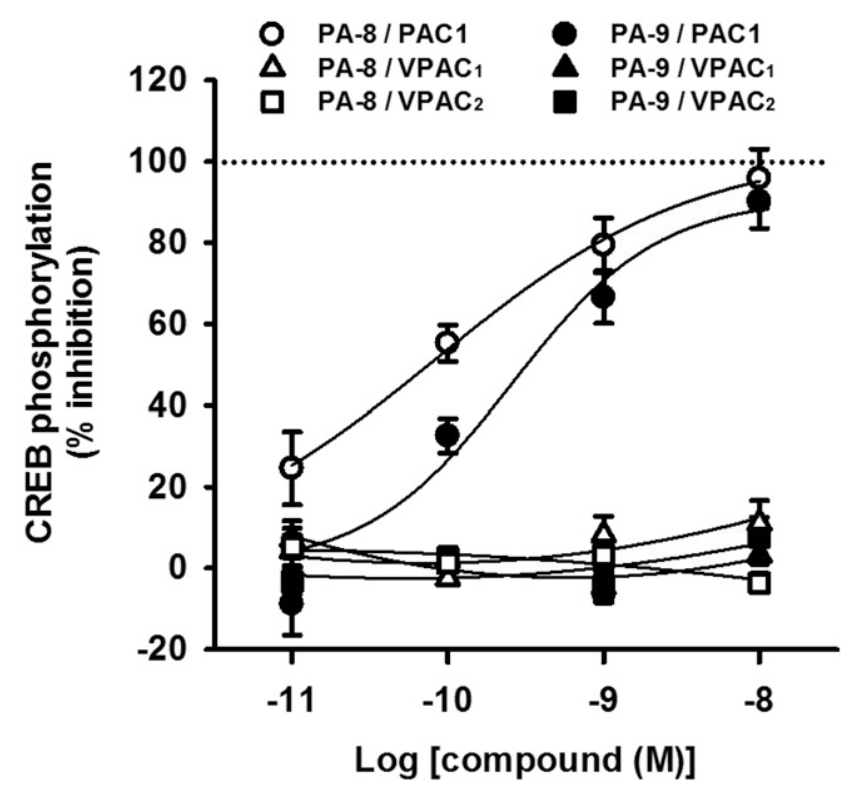

Fig. 4. Effects of PA-8 or PA-9 on the PACAP-induced phosphorylation of CREB in the CHO cells expressing PAC1, $\mathrm{VPAC}_{1}$, or $\mathrm{VPAC}_{2}$ receptors. The cells were incubated with PA-8 or PA-9 compounds (10 pM to $10 \mathrm{nM})$ for 30 minutes, then the cells were stimulated with PACAP $(1 \mathrm{nM})$. The data represent the mean \pm S.E.M. $(n=3-5)$.

(Fig. 6A). PA-8 and PA-10 almost completely suppressed PACAP-induced aversive responses. In contrast, a single intrathecal injection of PA-8, PA-9, or PA-10 (100 pmol/5 $\mu \mathrm{l})$ alone did not induce aversive responses, at least during the 30-minute observation period after injection (Fig. 6B), suggesting that these compounds do not have algesic effects.

Finally, we examined the effects of the three PA compounds on the induction of PACAP-induced mechanical allodynia. In accordance with our previous report (Yokai et al., 2016), a single intrathecal injection of PACAP (100 pmol) induced mechanical allodynia beginning 1 day after injection and persisting for a minimum of 28 days (Fig. 6C). Co-injection of PA-8, PA-9, or PA-10 (100 pmol each) with PACAP (100 pmol) significantly blocked the induction of PACAP-induced mechanical allodynia (Fig. 6C). However, a single intrathecal injection of PA-8, PA-9, or PA-10 (100 pmol) did not induce mechanical allodynia, at least during the 3-day observation period after injection (Fig. 6D).

Models of PA-8 or PA-9 Binding with PAC1 Receptor. Since the identified compounds, particularly PA-8 and PA-9, showed potent and specific antagonistic activity toward PAC1 receptors, we constructed the models of PA- 8 or PA-9 binding with the $N$-terminal EC domain of the PAC1 receptor (Fig. 7). Both PA-8 and PA-9 were found to be well accommodated in the subpocket formed by L80, F81, I83, G91, V92, P107, A112, and $\mathrm{C} 113$ of the $\mathrm{PAC} 1$ receptor, precipitating in hydrophobic interactions. In addition, PA- 8 was predicted to form two hydrogen bonds with the backbone carbonyl oxygen of S120 and the oxygen of the carboxylate group of E121 (Fig. 7A). PA-9 was also predicted to form three hydrogen bonds with the backbone carbonyl oxygens of L80, V92, and S120 (Fig. 7B).

\section{Discussion}

In the present study, we attempted to identify smallmolecule antagonists of the PAC1 receptor by using in silico screening. The 10 compounds identified were further

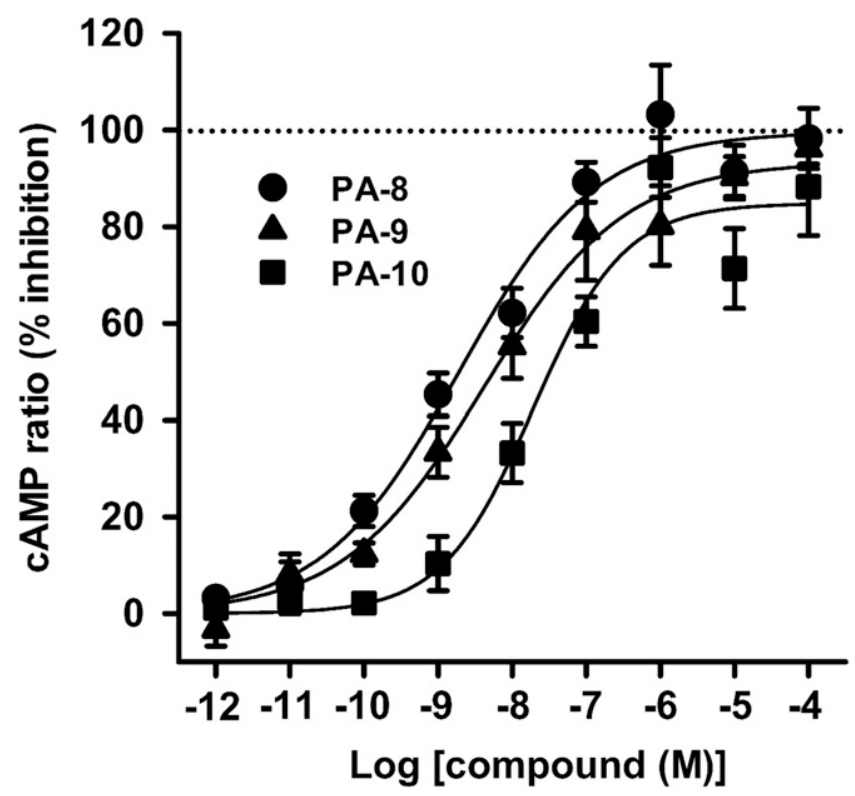

Fig. 5. Dose-dependent inhibitory effects of PA-8, PA-9, or PA-10 on the PACAP-induced cAMP elevation in the CHO cells expressing PAC1 receptors. The cells were incubated with PA-8, PA-9, or PA-10 (1 pM to $100 \mu \mathrm{M})$ for 30 minutes, then the cells were stimulated with PACAP $(1 \mathrm{nM})$ for 1 hour and assessed the level of cAMP using cAMP-Glo Assay kit. The data represent the mean \pm S.E.M. $(n=3-5)$.

characterized using cell-based functional assays: PACAPinduced CREB phosphorylation and cAMP elevation. We finally identified three small-molecules (named PA-8, PA-9, and PA-10) as candidate antagonists of the PAC1 receptor from 10 virtual hits, corresponding to a hit rate of $30 \%$. This high hit rate suggested that our multifilter virtual screening protocol was quite useful for exploring novel PAC1 receptor antagonists. The identified compounds, particularly PA-8 and PA-9, showed potent and specific antagonistic activity toward PAC1 receptors, but not $\mathrm{VPAC}_{1}$ or $\mathrm{VPAC}_{2}$ receptors, and no detectable agonistic activity toward PAC1 receptors. These compounds also blocked intrathecal PACAP-induced aversive responses and mechanical allodynia without any algesic effects. To the best of our knowledge, this is the first report on the discovery of nonpeptide small-molecule antagonists of the PAC1 receptor.

Studies of PACAP and PACAP receptors have been hampered due to a lack of specific PAC1 receptor antagonists. Although several PAC1 receptor antagonists, such as PACAP 6-38, M65, and max.d.4 (Robberecht et al., 1992; Uchida et al., 1998; Moro et al., 1999), have been often used as pharmacological tools, limitations exist in their use in vivo because all of them are peptide compounds. In particular, PACAP 6-38, a peptide fragment lacking the first five residues of PACAP, has been used in numerous studies as a PAC1 specific antagonist; however, the $\mathrm{IC}_{50}$ value of binding of PACAP 6-38 for PAC1 and $\mathrm{VPAC}_{2}$ receptors are 30 and $40 \mathrm{nM}$, respectively (Gourlet et al., 1995). Therefore, PACAP 6-38 should be recognized as a potent dual PAC1/VPAC ${ }_{2}$ antagonist (Laburthe et al., 2007). It has also been reported that PACAP 6-38 acts as an agonist in certain cells/tissues, including isolated rat trachea and trigeminal ganglia neurons (Nemeth et al., 2006; Reglodi et al., 2008; Sághy et al., 2015). Maxadilan, a peptide isolated from salivary gland extracts of the hematophagous sand fly, is a specific 

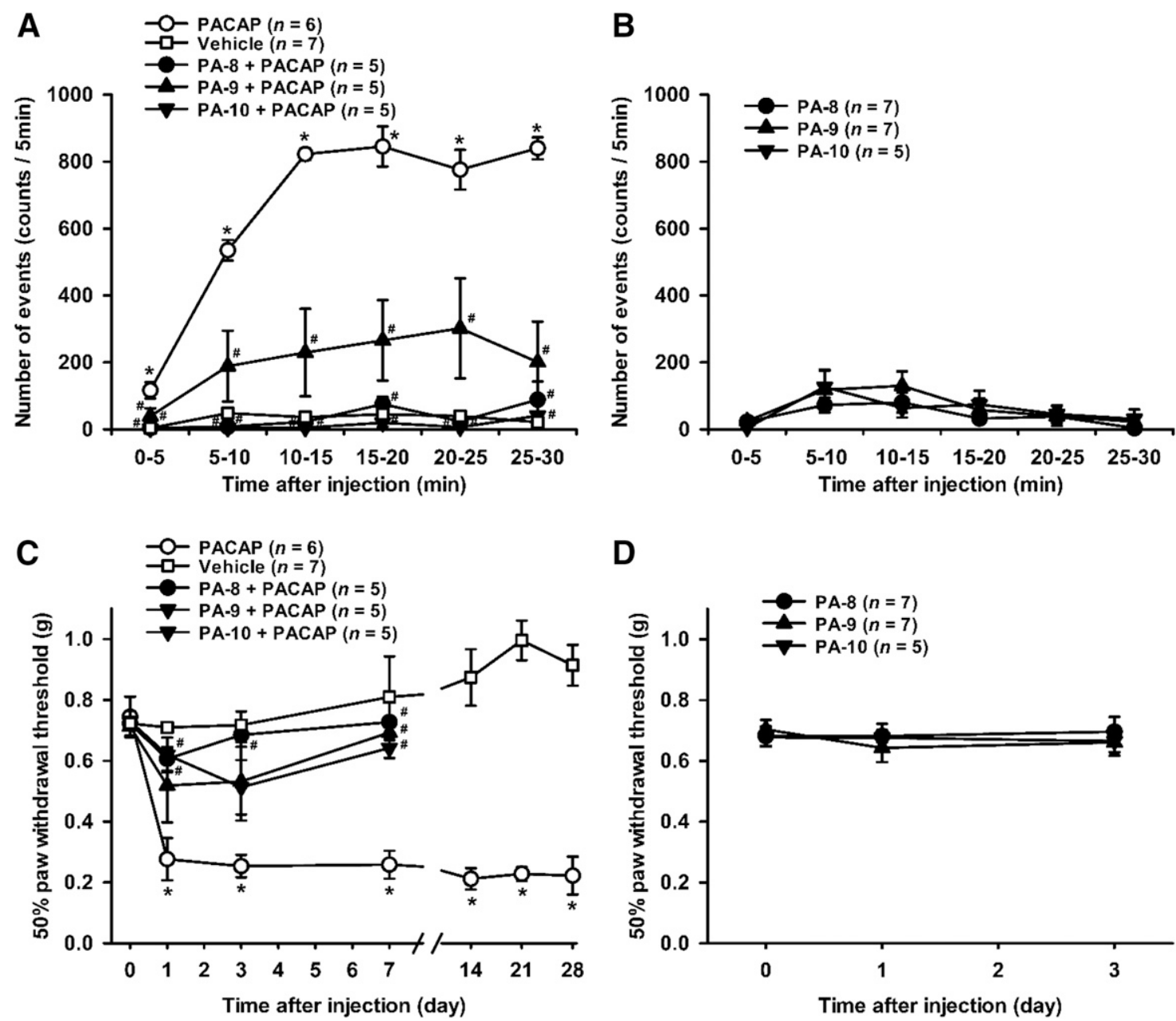

Fig. 6. Blockade of PACAP-induced aversive responses and mechanical allodynia by the identified PA compounds. (A) A single intrathecal injection of PACAP (100 pmol) induced transient aversive responses, and the PACAP-induced behaviors were ameliorated by co-injection of PA-8, PA-9, or PA-10 (100 pmol each). (B) PA-8, PA-9, or PA-10 (100 pmol each intrathecally) in itself did not induce aversive responses. (C) A single intrathecal injection of PACAP (100 pmol) induced long-lasting mechanical allodynia, and the induction of allodynia was prevented by co-injection of PA-8, PA-9, or PA-10 (100 pmol each). (D) PA-8, PA-9, or PA-10 (100 pmol each intrathecally) per se did not induce mechanical allodynia. $* P<0.05$ compared with vehicle. ${ }^{\#} P<0.05$ compared with PACAP.

agonist of the PAC1 receptor, and a truncated peptide (\#25-41) of maxadilan (termed M65) has been considered a specific PAC1 receptor antagonist (Uchida et al., 1998; Moro et al., 1999). However, M65 also showed similar stimulatory effects as maxadilan on trigeminal ganglia neurons (Sághy et al., 2015). The compounds we identified (PA-8, PA-9, and PA-10) antagonized PACAP-induced intracellular events in PAC1expressing cells but not in $\mathrm{VPAC}_{1^{-}}$or $\mathrm{VPAC}_{2}$-expressing cells at very low doses (nanomolar order), and they had no agonistic actions. These small-molecules are likely to contribute to advancing our understanding of the function of PACAP-PAC1 receptor systems.
A

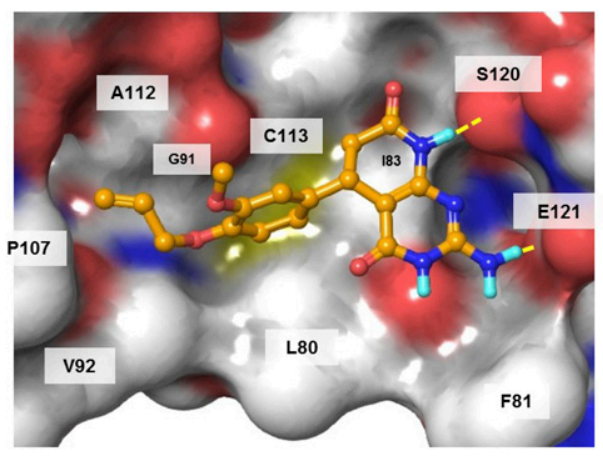

B

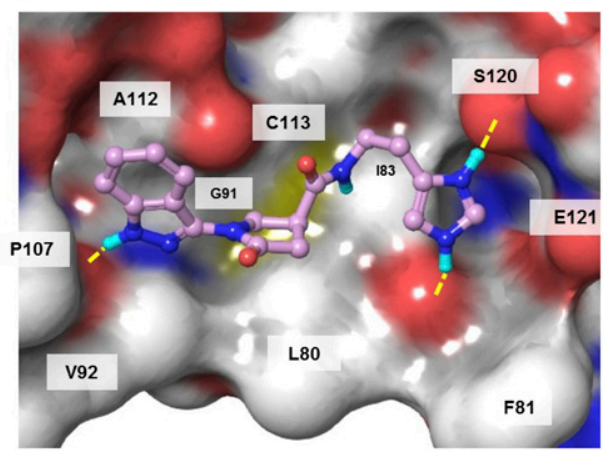

Fig. 7. Docking models of PA-8 (A) or PA-9 (B) with PAC1 receptor. Hydrogen-bonding interactions are indicated by yellow dashed lines. 
A growing body of evidence demonstrates that the PACAPPAC1 receptor signaling system in the spinal dorsal horn plays a crucial role in pain transmission. Recently, we showed that a single intrathecal administration of PACAP or maxadilan induced short-lasting aversive responses, such as licking, biting, and scratching, followed by a long-lasting mechanical allodynia (Ohnou et al., 2016; Yokai et al., 2016). Such aversive responses and mechanical allodynia were attenuated by intrathecal pretreatment with max.d.4, a specific PAC1 receptor antagonist (Ohnou et al., 2016; Yokai et al., 2016). In contrast to maxadilan, VIP, a selective agonist of $\mathrm{VPAC}_{1}$ and $\mathrm{VPAC}_{2}$ receptors but not PAC1 receptor, induced neither nociceptive behaviors nor mechanical allodynia (Ohnou et al., 2016; Yokai et al., 2016). Consistent with our previous findings, it has been reported that PAC1 receptor knockout mice have markedly reduced late-phase nociceptive responses in the formalin test (Jongsma et al., 2001). These findings strongly suggest that spinal PACAP-induced nociceptive behaviors and mechanical allodynia are predominantly mediated by the PAC1 receptor and that the PAC1 receptor is a useful target for this type of pain. In fact, in the present study, PACAP-induced aversive behaviors and mechanical allodynia were significantly inhibited by PA-8, PA-9, and PA-10. The results thus indicate that the identified compounds may be effective against pain in which the PACAP-PAC1 receptor system is implicated. It has been reported that transection of the sciatic nerve induces a rapid and strong upregulation of PACAP mRNA levels in mice and rats (Zhang et al., 1995, 1996; Mabuchi et al., 2004). Upregulation of PACAP in the dorsal root ganglia was also observed after peripheral inflammation induced by complete Freund's adjuvant (Zhang et al., 1998). Development of both inflammatory and neuropathic pain was shown to be abrogated in PACAP-deficient mice (Mabuchi et al., 2004). These results indicate that the spinal PACAP-PAC1 receptor system plays a central role in the regulation of these types of inflammatory and neuropathic pain, and potentially other types, and that the drugs targeting the PAC1 receptor may provide a new approach to treat PACAP-PAC1-mediated pain. Whether the novel PAC1 receptor antagonists identified here are effective against inflammatory and/or neuropathic pain needs to be examined in future studies.

Recent studies also point to the involvement of the PACAP$\mathrm{PAC} 1$ receptor system in migraine pain and neuropsychiatric disorders. It has been reported that the infusion of PACAP, but not VIP, elicits a migraine-like attack in some healthy volunteers and migraine patients (Hansen et al., 2006; Rahmann et al., 2008; Schytz et al., 2009). In rodents, nitroglycerin-induced light-aversive behaviors (photophobia), one of the most common symptoms of migraine, were significantly reduced in PACAP knockout mice (Markovics et al., 2012). Thus, current evidence suggests the involvement of PACAP-PAC1 receptor signaling in migraine pathophysiology and the PAC1 receptor as a target for migraine treatment. A role of the PACAP-PAC1 receptor system in anxiety- and stress-related behaviors has also been proposed. It has been reported that chronic stress in mice leads to the upregulation of PACAP mRNA in the bed nucleus of the stria terminalis (BNST) (Hammack et al., 2009; Roman et al., 2014). Microinjection of PACAP or maxadilan into the BNST induced anxiety-like behaviors, and PACAP 6-38 injection into the BNST blocked stress-induced psychiatric behaviors
(Roman et al., 2014). Furthermore, mice lacking PACAP or the PAC1 receptor have been found to demonstrate reduced anxiety-like behavior (Hashimoto et al., 2001; Otto et al., 2001; Hattori et al., 2012). Whether the new PAC1 receptor antagonists we identified suppress migraine-like and/or stress-associated behaviors in mice is an interesting question that should be examined in future studies.

In conclusion, we identified novel small-molecule antagonists of the PAC1 receptor using in silico screening and in vitro/vivo pharmacological assays. These compounds will be useful for elucidating new functional roles of PAC1 receptor systems in the peripheral and central nervous systems and for further development of novel drugs against neuropathic and/ or inflammatory pain, migraine, and psychiatric disorders.

\section{Acknowledgments}

We thank Dr. Nobuyuki Kurosawa and Dr. Masaharu Isobe (University of Toyama) for providing LAS-1000 plus and Dr. Shigenori Kawahara and Koji Usui (University of Toyama) for providing animal breeding facility. We also thank all the staff members of the Institute of Laboratory Animal Science Research Support Center, Kagoshima University.

\section{Authorship Contributions}

Participated in research design: Takasaki, Toyooka, Miyata, Kurihara.

Conducted experiments: Takasaki, A. Watanabe, Yokai, Nagashima, Okada, Fukuchi, Kurihara.

Contributed new reagents or analytic tools: Y. Watanabe, Hayakawa, Gouda.

Performed data analysis: Takasaki, A. Watanabe, Yokai, Y. Watanabe, Hayakawa, Gouda, Kurihara.

Wrote or contributed to the writing of the manuscript: Takasaki, Toyooka, Miyata, Gouda, Kurihara.

\section{References}

Chaplan SR, Bach FW, Pogrel JW, Chung JM, and Yaksh TL (1994) Quantitative assessment of tactile allodynia in the rat paw. J Neurosci Methods 53:55-63.

Davis-Taber R, Baker S, Lehto SG, Zhong C, Surowy CS, Faltynek CR, Scott VE and Honore P (2008) Central pituitary adenylate cyclase 1 receptors modulate nociceptive behaviors in both inflammatory and neuropathic pain states. J Pain 9: $449-456$

Dickson L and Finlayson K (2009) VPAC and PAC receptors: from ligands to function. Pharmacol Ther 121:294-316.

Duan J, Dixon SL, Lowrie JF, and Sherman W (2010) Analysis and comparison of 2D fingerprints: insights into database screening performance using eight fingerprint methods. J Mol Graph Model 29:157-170.

Dun NJ, Miyazaki T, Tang H, and Dun EC (1996) Pituitary adenylate cyclase activating polypeptide immunoreactivity in the rat spinal cord and medulla: implication of sensory and autonomic functions. Neuroscience 73:677-686.

Gourlet P, Vandermeers A, Vandermeers-Piret MC, Rathé J, De Neef P, and Robberecht P (1995) Fragments of pituitary adenylate cyclase activating polypeptide discriminate between type I and II recombinant receptors. Eur $J$ Pharmacol 287:7-11.

Hammack SE, Cheung J, Rhodes KM, Schutz KC, Falls WA, Braas KM, and May V (2009) Chronic stress increases pituitary adenylate cyclase-activating peptide (PACAP) and brain-derived neurotrophic factor (BDNF) mRNA expression in the bed nucleus of the stria terminalis (BNST): roles for PACAP in anxiety-like behavior. Psychoneuroendocrinology 34:833-843.

Hansen JM, Sitarz J, Birk S, Rahmann AM, Oturai PS, Fahrenkrug J, Olesen J, and Ashina M (2006) Vasoactive intestinal polypeptide evokes only a minimal headache in healthy volunteers. Cephalalgia 26:992-1003.

Hashimoto H, Shintani N, Tanaka K, Mori W, Hirose M, Matsuda T, Sakaue M, Miyazaki J, Niwa H, Tashiro F, et al. (2001) Altered psychomotor behaviors in mice lacking pituitary adenylate cyclase-activating polypeptide (PACAP). Proc Natl Acad Sci USA 98:13355-13360.

Hattori S, Takao K, Tanda K, Toyama K, Shintani N, Baba A, Hashimoto H, and Miyakawa T (2012) Comprehensive behavioral analysis of pituitary adenylate cyclase-activating polypeptide (PACAP) knockout mice. Front Behav Neurosci 6:58.

Hylden JL and Wilcox GL (1980) Intrathecal morphine in mice: a new technique. Eur J Pharmacol 67:313-316.

Jongsma H, Pettersson LM, Zhang Yz, Reimer MK, Kanje M, Waldenström A, Sundler F, and Danielsen N (2001) Markedly reduced chronic nociceptive response in mice lacking the PAC1 receptor. Neuroreport 12:2215-2219.

Jongsma Wallin H, Pettersson LM, Verge VM, and Danielsen N (2003) Effect of anti-nerve growth factor treatment on pituitary adenylate cyclase activating 
polypeptide expression in adult sensory neurons exposed to adjuvant induced inflammation. Neuroscience 120:325-331.

Laburthe M, Couvineau A, and Tan V (2007) Class II G protein-coupled receptors for VIP and PACAP: structure, models of activation and pharmacology. Peptides 28 : 1631-1639.

Mabuchi T, Shintani N, Matsumura S, Okuda-Ashitaka E, Hashimoto H, Muratani T, Minami T, Baba A, and Ito S (2004) Pituitary adenylate cyclase-activating polypeptide is required for the development of spinal sensitization and induction of neuropathic pain. J Neurosci 24:7283-7291.

Markovics A, Kormos V, Gaszner B, Lashgarara A, Szoke E, Sandor K, Szabadfi K, Tuka B, Tajti J, Szolcsanyi J, et al. (2012) Pituitary adenylate cyclase-activating polypeptide plays a key role in nitroglycerol-induced trigeminovascular activation in mice. Neurobiol Dis 45:633-644.

Miyata A, Arimura A, Dahl RR, Minamino N, Uehara A, Jiang L, Culler MD, and Coy DH (1989) Isolation of a novel 38 residue-hypothalamic polypeptide which stimulates adenylate cyclase in pituitary cells. Biochem Biophys Res Commun 164: 567-574.

Miyata A, Jiang L, Dahl RD, Kitada C, Kubo K, Fujino M, Minamino N, and Arimura A (1990) Isolation of a neuropeptide corresponding to the $\mathrm{N}$-terminal 27 residues of the pituitary adenylate cyclase activating polypeptide with 38 residues (PACAP38). Biochem Biophys Res Commun 170:643-648.

Moller K, Zhang YZ, Håkanson R, Luts A, Sjölund B, Uddman R, and Sundler F (1993) Pituitary adenylate cyclase activating peptide is a sensory neuropeptide: immunocytochemical and immunochemical evidence. Neuroscience 57:725-732.

Moro O, Wakita K, Ohnuma M, Denda S, Lerner EA, and Tajima M (1999) Functional characterization of structural alterations in the sequence of the vasodilatory peptide maxadilan yields a pituitary adenylate cyclase-activating peptide type 1 receptor-specific antagonist. J Biol Chem 274:23103-23110.

Mulder H, Uddman R, Moller K, Zhang YZ, Ekblad E, Alumets J, and Sundler F (1994) Pituitary adenylate cyclase activating polypeptide expression in sensory neurons. Neuroscience 63:307-312.

Narita M, Dun SL, Dun NJ, and Tseng LF (1996) Hyperalgesia induced by pituitary adenylate cyclase-activating polypeptide in the mouse spinal cord. Eur J Pharmacol 311:121-126.

Németh J, Reglödi D, Pozsgai G, Szabó A, Elekes K, Pintér E, Szolcsányi J, and Helyes Z (2006) Effect of pituitary adenylate cyclase activating polypeptide-38 on sensory neuropeptide release and neurogenic inflammation in rats and mice. Neuroscience 143:223-230.

Ohnou T, Yokai M, Kurihara T, Hasegawa-Moriyama M, Shimizu T, Inoue K, Kambe Y, Kanmura Y, and Miyata A (2016) Pituitary adenylate cyclase-activating polypeptide type 1 receptor signaling evokes long-lasting nociceptive behaviors through the activation of spinal astrocytes in mice. J Pharmacol Sci 130:194-203.

Ohsawa M, Brailoiu GC, Shiraki M, Dun NJ, Paul K, and Tseng LF (2002) Modulation of nociceptive transmission by pituitary adenylate cyclase activating polypeptide in the spinal cord of the mouse. Pain 100:27-34.

Oprea TI, Davis AM, Teague SJ, and Leeson PD (2001) Is there a difference between leads and drugs? A historical perspective. J Chem Inf Comput Sci 41:1308-1315.

Otto C, Martin M, Wolfer DP, Lipp HP, Maldonado R, and Schütz G (2001) Altered emotional behavior in PACAP-type-I-receptor-deficient mice. Brain Res Mol Brain Res 92:78-84.

Rahmann A, Wienecke T, Hansen JM, Fahrenkrug J, Olesen J, and Ashina M (2008) Vasoactive intestinal peptide causes marked cephalic vasodilation, but does not induce migraine. Cephalalgia 28:226-236.

Reglodi D, Borzsei R, Bagoly T, Boronkai A, Racz B, Tamas A, Kiss P, Horvath G Brubel R, Nemeth J, et al. (2008) Agonistic behavior of PACAP6-38 on sensory nerve terminals and cytotrophoblast cells. J Mol Neurosci 36:270-278.

Robberecht P, Gourlet P, De Neef P, Woussen-Colle MC, Vandermeers-Piret MC, Vandermeers A, and Christophe J (1992) Structural requirements for the occupancy of pituitary adenylate-cyclase-activating-peptide (PACAP) receptors and adenylate cyclase activation in human neuroblastoma NB-OK-1 cell membranes. Discovery of PACAP(6-38) as a potent antagonist. Eur J Biochem 207:239-246.
Roman CW, Lezak KR, Hartsock MJ, Falls WA, Braas KM, Howard AB, Hammack SE, and May V (2014) PAC1 receptor antagonism in the bed nucleus of the stria terminalis (BNST) attenuates the endocrine and behavioral consequences of chronic stress. Psychoneuroendocrinology 47:151-165.

Sághy É, Payrits M, Helyes Z, Reglődi D, Bánki E, Tóth G, Couvineau A, and Szőke É (2015) Stimulatory effect of pituitary adenylate cyclase-activating polypeptide 6-38, M65 and vasoactive intestinal polypeptide 6-28 on trigeminal sensory neurons. Neuroscience 308:144-156.

Sakashita Y, Kurihara T, Uchida D, Tatsuno I, and Yamamoto T (2001) Involvement of PACAP receptor in primary afferent fibre-evoked responses of ventral roots in the neonatal rat spinal cord. Br J Pharmacol 132:1769-1776.

Sándor K, Kormos V, Botz B, Imreh A, Bölcskei K, Gaszner B, Markovics A, Szolcsányi J, Shintani N, Hashimoto H, et al. (2010) Impaired nocifensive behaviours and mechanical hyperalgesia, but enhanced thermal allodynia in pituitary adenylate cyclase-activating polypeptide deficient mice. Neuropeptides 44:363-371.

Sastry M, Lowrie JF, Dixon SL, and Sherman W (2010) Large-scale systematic analysis of $2 \mathrm{D}$ fingerprint methods and parameters to improve virtual screening enrichments. J Chem Inf Model 50:771-784.

Schytz HW, Birk S, Wienecke T, Kruuse C, Olesen J, and Ashina M (2009) PACAP38 induces migraine-like attacks in patients with migraine without aura. Brain 132: $16-25$.

Shimizu T, Katahira M, Sugawara H, Inoue K, and Miyata A (2004) Diverse effects of intrathecal pituitary adenylate cyclase-activating polypeptide on nociceptive transmission in mice spinal cord. Regul Pept 123:117-122.

Sun C, Song D, Davis-Taber RA, Barrett LW, Scott VE, Richardson PL, Pereda-Lopez A, Uchic ME, Solomon LR, Lake MR, et al. (2007) Solution structure and mutational analysis of pituitary adenylate cyclase-activating polypeptide binding to the extracellular domain of PAC1-RS. Proc Natl Acad Sci USA 104:7875-7880.

Uchida D, Tatsuno I, Tanaka T, Hirai A, Saito Y, Moro O, and Tajima M (1998) Maxadilan is a specific agonist and its deleted peptide (M65) is a specific antagonist for PACAP type 1 receptor. Ann N Y Acad Sci 865:253-258.

Vaudry D, Falluel-Morel A, Bourgault S, Basille M, Burel D, Wurtz O, Fournier A Chow BK, Hashimoto H, Galas L, et al. (2009) Pituitary adenylate cyclaseactivating polypeptide and its receptors: 20 years after the discovery. Pharmacol Rev 61:283-357.

Yokai M, Kurihara T, and Miyata A (2016) Spinal astrocytic activation contributes to both induction and maintenance of pituitary adenylate cyclase-activating polypeptide type 1 receptor-induced long-lasting mechanical allodynia in mice. Mol Pain 12:1-12.

Zhang Q, Shi TJ, Ji RR, Zhang YZ, Sundler F, Hannibal J, Fahrenkrug J, and Hökfelt $\mathrm{T}$ (1995) Expression of pituitary adenylate cyclase-activating polypeptide in dorsal root ganglia following axotomy: time course and coexistence. Brain Res 705: 149-158.

Zhang Y, Danielsen N, Sundler F, and Mulder H (1998) Pituitary adenylate cyclaseactivating peptide is upregulated in sensory neurons by inflammation. Neuroreport 9:2833-2836.

Zhang Y, Malmberg AB, Yaksh TL, Sjölund B, Sundler F, and Håkanson R (1997) Capsaicin-evoked release of pituitary adenylate cyclase activating peptide (PACAP) and calcitonin gene-related peptide (CGRP) from rat spinal cord in vivo. Regul Pept 69:83-87.

Zhang YZ, Hannibal J, Zhao Q, Moller K, Danielsen N, Fahrenkrug J, and Sundler F (1996) Pituitary adenylate cyclase activating peptide expression in the rat dorsal root ganglia: up-regulation after peripheral nerve injury. Neuroscience $\mathbf{7 4}$ : 1099-1110.Zimmermann M (1983) Ethical guidelines for investigations of experimental pain in conscious animals. Pain 16:109-110.

Address correspondence to: Dr. Ichiro Takasaki, Department of Pharmacology, Graduate School of Science and Engineering, University of Toyama, Toyama 930-8555, Japan. E-mail: takasaki@eng.u-toyama.ac.jp 